\title{
Numerical Simulations in Astrophysics: Supernovae Explosions, Magnetorotational Model and Neutrino Emission
}

\author{
G.S. BISNOVATYI-KOGAN \\ Space Research Institute, Russian Academy of Sciences, Moscow, Russia
}

(Received 1 March 1999)

\begin{abstract}
Theories of stellar evolution and stellar explosion are based on results of numerical simulations and even qualitative results are not available to get analytically. Supernovae are the last stage in the evolution of massive stars, following the onset of instability, collapse and formation of a neutron star. Formation of a neutron star is accompanied by a huge amount of energy, approximately $20 \%$ of the rest mass energy of the star, but almost all this energy is released in the form of weakly interacting and hardly registrated neutrino. About $0.1 \%$ of the released neutrino energy would be enough for producing a supernovae explosion, but even transformation of such a small part of the neutrino energy into the kinetic energy of matter meets serious problems. Two variants are investigated for obtaining explosion. The first one is based on development of convective instability, and more effective heating of the outer layers by a neutrino flux.

The second model is based on transformation of a rotational energy of a rapidly rotating neutron star with its envelope into the energy of explosion due to action of a magnetic field as a transformation mechanism. Calculations in this model in 1- and 2-dimensions give a stable value of transformation of the rotational energy into the energy of explosion on the level of few percents. This occurrance to be enough for explanation of the energy release in supernova explosion. The last model gives a direct demonstration of nonlinear interaction between hydrodynamical and hydromagnetic systems. At first a field is amplified by differential rotation, then this enhanced field leads to transformation of the rotational energy into the energy of explosion.
\end{abstract}

Keywords: Supernovae explosions, Magnetic fields, Neutrino emission

\section{INTRODUCTION}

A first understanding of the evolution of the Sun appeared after numerical calculations of Martin Schwarzschild made by in 1941 (see [31]). Twenty-five years later first numerical calculations of supernovae (SN) explosion have been performed
[14], revealing qualitative as well as quantitative features of this explosion: prevailing role of neutrino energy losses and big sensitivity to the physical input parameters.

A SN explosion is the end of the life of most massive stars with $M>8 M_{\odot}$. The flash itself results either from the thermal instability development in 
degenerate core, or from gravitational and partly nuclear energy release during collapse which leads to the neutron star formation. The rotation and magnetic field may play an important role in conversion of gravitational energy into energy of observable flash. A small part of stars (the most massive ones) seems to end their life with collapse and black hole formation. The collapse in this case may be "silent" and not lead to SN explosion. The physical processes accompanying $\mathrm{SN}$ explosions are: nuclear reactions, neutrino processes, convection; equation of state of matter in wide region of parameters, where effects of degeneracy and relativistic corrections are important, is described in the book [7]. Despite many efforts, the SN theory is far from complete even in spherically symmetrical approximation by reason of serious numeric and fundamental difficulties related to nonstationary convection, neutrino transport and equation of state for matter of a density above the nuclear.

\section{NUMERICAL CALCULATIONS OF SN IN THE HYDRODYNAMICAL MODEL}

Iron cores with mass $M_{\mathrm{Fe}} \geq 1.4 M_{\odot}$ lose their stability through the iron dissociation which directly leads to a rapid collapse. The iron core forms in stars with initial mass $M_{\mathrm{i}}>10 M_{\odot}$, while for $M>13 M_{\odot}$ all stages of nuclear burning proceed smoothly. As all evolutionary calculations yield significant uncertainty in the relationship $M_{\mathrm{Fe}}\left(M_{\mathrm{i}}\right)$, the stability loss due to the iron dissociation is certain for single stars with $M_{\mathrm{i}}>13 \pm 3 M_{\odot}$.

Hydrodynamical calculations of iron core collapse have been first performed in [14], and soon after in $[5,18]$. Hydrodynamical equations have been solved and, massive stellar cores $\left(M \geq 2 M_{\odot}\right)$ on the boundary of hydrodynamical stability have been taken for initial conditions. These studies take into account formation of electron and muon neutrinos during collapse, the role of neutrino in the envelope heating and probable ejection, and the effect of burning of thermonuclear fuel ${ }^{12} \mathrm{C},{ }^{16} \mathrm{O}$ remaining around the iron core. It has been noted in [10] that the reflection of infalling matter from the surface of stable neutron star and formation of a shock wave (bounce) may also be important for producing $\mathrm{SN}$ explosion. Numerous calculations to date (see reviews $[11,16,17,36,37]$ ) have revealed sensitivity of the results to the equation of state of nuclear matter, quantity of remaining thermonuclear fuel, treatment of convection. The results are strongly influenced by adopted methods for including neutrinos at transparent and opaque stages. Calculations including neutrino processes in a self-consistent way over the entire star were first performed by Nadyozhin $[26,27]$. It was shown that neutrinos are strongly damping the matter inflow, preventing the bounce, and their heating of matter (neutrino deposition) is not enough for a formation of a strong shock and explosion. The mean energies of neutrinos arising in collapse are $\sim 10 \mathrm{MeV} .^{\dagger}$ Inclusion of thermonuclear burning of oxygen in the envelope, muon- and tau-neutrinos, momentum transfer from neutrinos to nuclei caused by coherent scattering due to neutral currents does almost not alter the results.

\subsection{Neutrino Convection in Collapsing Stellar Cores: 2D and 3D Calculations}

Neutrino flux is formed in the neutrino opaque core and goes out freely over the neutrinosphere, where the optical depth to the neutrino absorption is about unity. Huge temperature and lepton concentration gradients are developed in the vicinity of the neutrinosphere, leading to convective instability. Convective motions in the neutrinosphere might bring hot material outward and increase the mean energy and flux of escaping neutrinos whose deposition could initiate an explosion.

An unstable lepton and entropy profiles, formed after $\sim 10 \mathrm{~ms}$ of the creation of shock wave and

\footnotetext{
${ }^{\dagger}$ In [23], the mean energies of electron neutrinos equal $14 \mathrm{MeV}$ electron antineutrinos, $15 \mathrm{MeV}$, other neutrino species $\left(\nu_{\mu}, \tilde{\nu}_{\mu}, \nu_{\tau}, \tilde{\nu}_{\tau}\right)$, $32 \mathrm{MeV}$. The total energy of emitted neutrinos $\sim 6 \times 10^{53} \mathrm{erg}$ is distributed almost equally between these six types of particles.
} 
bounce of the core, can drive a violent ReyleighTaylor-like overturn studied in [12]. Coupling 2-D hydrodynamics treated by precise-parabolic method (PPM), with independent 1-D neutrino transport was used. The explosion was obtained in this model, while without neutrino transport, or with account of convection in 1-D hydrodynamical model the explosion did not happen. Extended calculations in similar model with 2-D neutrino transport have been presented in [13]. Here convection becomes so violent, that spherical and even plane symmetry of the core are strongly broken, neutrino emission and mass ejection goes anisotropically, inducing the explosion with ejection of few tens of high entropy clumps, and giving a kick to a neutron star, which by estimations can reach a speed of $\sim 500 \mathrm{~km} / \mathrm{s}$.

3-D simulations of convection in the shocked matter of the SN core have been done in [32], assuming that the neutrino radiation from the proto neutron star is radial, but axisymmetric. The asphericity of the neutrino flux was connected with rapid rotation of the protoneutron star. The formation of high-entropy hot bubbles and jet-like explosion was obtained as a result, but explosion energy problem was not considered.

PPM method was used in 2-D calculations $[19,20]$ of neutrino-driven $\mathrm{SN}$ with convective overturn and accretion. The effects of convection obtained here are less pronounced than in [13], while powerful explosion is obtained in a certain, although rather narrow, window of core $\nu$ fluxes in which 1$\mathrm{D}$ models do not explode. The maximum attainable velocities of the kick are estimated to be around $200 \mathrm{~km} / \mathrm{s}$.

Extensive 2-D study of SN explosion following the collapse of cores of two massive stars (15 and $\left.25 M_{\odot}\right)$ have been performed in [15]. The calculations begin at the onset of core collapse and stop several hundred milliseconds after the bounce, at which time successful explosion of appropriate magnitude has been obtained. The explosion is powered by the heating of the envelope due to neutrino emitted by the protoneutron star. This heating generates strong convection outside the neutrinosphere which was demonstrated to be critical to the explosion. Convection leads to violation of radiative equilibrium between neutrino emission and absorption. Thus explosions become quite insensitive to the physical input parameters, such as neutrino cross-section or nuclear equation of state parameter.

Smooth particle hydrodynamics (SPH) code was used for 2-D calculations with spherically symmetric gravity and realistic equation of state. 2-D explicit code for neutrino transport was developed with account of most important processes of neutrino emission, absorption and scattering. A peculiar characterictic of neutrino processes in $\mathrm{SN}$ is that dominant process which leads to neutrino trapping does not affect the neutrino spectrum, because elastic scattering between nucleons and neutrinos happens almost without the energy exchange. That leads to the situation, where the optical depth can be large without thermalizing the fields. As a result, local thermodynamic equilibrium cannot be assumed.

The main features common to all 2-D simulations made in [15] are following. After an initial period of dynamical infall lasting a few hundred milliseconds, the central density becomes supernuclear, the core hardens and a bounce shock is launched. Within a few milliseconds this shock stalls due to energy losses at a radius $\sim 150 \mathrm{~km}$. At this point 2-D calculations begin to differ greatly from 1-D computations because of the onset of hydrodynamical instabilities. Most important to the $\mathrm{SN}$ is the neutrino-driven convection that lasts for over $100 \mathrm{~ms}$ until a successful explosion is achieved.

Investigations of collapse and explosion of rotating cores have shown that the explosion proceeds in the same manner as in the nonrotating case, except the rotation has a strong influence on the shape of the convective patterns that develope above the protoneutron star. As was pointed in [15], the most wanting aspect of the calculations remains in neutrino physics, because of the obvious difficulties of radiation transport in multidimensions. The basic flux-limited approximation leaves much to be desired in nearly optically thin regions, and 
also nonelastic neutrino nucleus interactions have been ignored, which could play an important role in the explosion. As was indicated in [15], at a time $\sim 200 \mathrm{~ms}$ SPH model ceased to adequately resolve the atmosphere above the neutron star and simulations had to be stopped. The fact the this time is close to the time of the formation of the successful explosion shock makes it desirable to check more carefully the role of numerical effects in these calculations. Another variant of 2-D calculations with similar input physics, but different numerical scheme and initial conditions, have been performed in [25], and no explosion was obtained in presence of the neutrino-driven convection.

\section{NUMERICAL SIMULATIONS OF THE MAGNETOROTATIONAL MODEL OF SUPERNOVAE EXPLOSION}

When all the above mechanisms of explosion prove to be inefficient, the magnetic field may convert the rotational energy of a neutron star resulting from collapse into kinetic energy of the envelope and thus ensures a SN explosion. A magnetorotational model of explosion has been suggested in [6]. Numerical calculations for this model have been made to cylindrical approximation in $[1,10]$, spherically symmetric approximation in [24] and in a simplified 2-D formulation in [30]. The results of these calculations are in qualitative agreement and give a conversion of $\sim 3 \%$ of the rotational energy into kinetic energy of the outburst. For $E_{\mathrm{rot}}=$ $10^{53}$ erg we have the energy $E_{\text {kin }}=3 \times 10^{51}$ erg sufficient to account for a $\mathrm{SN}$ explosion.

\subsection{Qualitative Description of Magnetorotational Explosion}

When a rapidly rotating presupernova collapses, it leads to formation of a rapidly rotating neutron star surrounded by a differentially rotating envelope in which the centrifugal forces are comparable with gravitational ones. The differential rotation twists the lines of magnetic force, thereby causing the magnetic field with initial energy $\epsilon_{\mathrm{M}} \ll \epsilon_{\mathrm{G}}$ to increase linearly in time. When the energy of the field in the envelope approaches $\epsilon_{\mathrm{M}} \sim \epsilon_{\mathrm{G}}$, the magnetic pressure pushes the material outward. The arising wave of compression propagates over medium with falling density, gets enhanced and transforms into shock to result in a powerful explosion. As the compression wave and subsequent shock move outward, their energy keeps increasing, maintained by rotational energy supplied by magnetic field. The magnetic field serves also to transfer to outer layers an essential part of the total angular momentum. Calculations of collapse for rotating star with a strong initial magnetic field have been done in [21]. The obtained picture of an explosion in the form of outbursts lined up along the dipole axis differs from the magnetorotational explosion where the major part of the outburst occurs in the equatorial plane. It is connected also with a choice of the initial magnetic field configuration, having maximum in the envelope, and zero radial component at the equator. The initial magnetic fields in [21] exceed 3-4 orders of magnitude of the really observed ones.

\subsection{Basic Equations of 2-D Model}

The equations of magnetohydrodynamics (MHD) with gravitation in cylindrical Eulerian system $(r, \varphi, z)$ at an infinite conductivity and axial symmetry $\partial / \partial \varphi=0$ read [7]

$$
\begin{aligned}
\frac{\partial v_{r}}{\partial t} & +v_{r} \frac{\partial v_{r}}{\partial r}+v_{z} \frac{\partial v_{r}}{\partial z}-\frac{v_{\varphi}^{2}}{r} \\
& =-\frac{1}{\rho} \frac{\partial P}{\partial r}-\frac{\partial \varphi_{\mathrm{G}}}{\partial r}+\frac{1}{\rho c}\left(j_{\varphi} B_{z}-j_{z} B_{\varphi}\right),
\end{aligned}
$$

$$
\frac{\partial v_{\varphi}}{\partial t}+v_{r} \frac{\partial v_{\varphi}}{\partial r}+v_{z} \frac{\partial v_{\varphi}}{\partial z}+\frac{v_{r} v_{\varphi}}{r}=\frac{1}{\rho c}\left(j_{z} B_{r}-j_{r} B_{z}\right),
$$

\footnotetext{
${ }^{\ddagger}$ Observations of radio emission at a relatively early period of the flash, roughly one year after the light peak, provide an indirect evidence for an essential role played by the magnetic field in supernova explosion [35].
} 


$$
\begin{gathered}
\frac{\partial v_{z}}{\partial t}+v_{r} \frac{\partial v_{z}}{\partial r}+v_{z} \frac{\partial v_{z}}{\partial z} \\
=-\frac{1}{\rho} \frac{\partial P}{\partial z}-\frac{\partial \varphi_{\mathrm{G}}}{\partial z}+\frac{1}{\rho c}\left(j_{r} B_{\varphi}-j_{\varphi} B_{r}\right), \\
\frac{\partial \rho}{\partial t}+v_{r} \frac{\partial \rho}{\partial r}+v_{z} \frac{\partial \rho}{\partial z}+\rho\left[\frac{1}{r} \frac{\partial\left(r v_{r}\right)}{\partial r}+\frac{\partial v_{z}}{\partial z}\right]=0, \\
\frac{\partial B_{r}}{\partial t}=-\frac{\partial}{\partial z}\left(v_{z} B_{r}-v_{r} B_{z}\right),
\end{gathered}
$$$$
\frac{\partial B_{\varphi}}{\partial t}=\frac{\partial}{\partial z}\left(v_{\varphi} B_{z}-v_{z} B_{\varphi}\right)-\frac{\partial}{\partial r}\left(v_{r} B_{\varphi}-v_{\varphi} B_{r}\right),
$$$$
\frac{\partial B_{z}}{\partial t}=\frac{1}{r} \frac{\partial}{\partial r}\left[r\left(v_{z} B_{r}-v_{r} B_{z}\right)\right]
$$$$
\frac{1}{r} \frac{\partial}{\partial r}\left(r B_{r}\right)+\frac{\partial B_{z}}{\partial z}=0
$$$$
j_{r}=-\frac{c}{4 \pi} \frac{\partial B_{\varphi}}{\partial z}
$$$$
j_{\varphi}=\frac{c}{4 \pi}\left(\frac{\partial B_{r}}{\partial z}-\frac{\partial B_{z}}{\partial r}\right)
$$$$
j_{z}=\frac{c}{4 \pi r} \frac{\partial}{\partial r}\left(r B_{\varphi}\right)
$$$$
\frac{1}{r} \frac{\partial}{\partial r}\left(r \frac{\partial \varphi_{\mathrm{G}}}{\partial r}\right)+\frac{\partial^{2} \varphi_{\mathrm{G}}}{\partial z^{2}}=4 \pi G \rho
$$

$$
\frac{\partial E}{\partial t}+v_{r} \frac{\partial E}{\partial r}+v_{z} \frac{\partial E}{\partial z}+\frac{P}{\rho}\left[\frac{1}{r} \frac{\partial\left(r v_{r}\right)}{\partial r}+\frac{\partial v_{z}}{\partial z}\right]=-f_{v}
$$

$$
P=P(\rho, T), \quad E=E(\rho, T), \quad f_{\nu}=f_{\nu}(\rho, T) .
$$

Here Equations (1)-(3) are the equations of motion with magnetic fields, (4) is the continuity equation, (5)-(7) are the "frozen-in" field equations $(\partial \vec{B} / \partial t=\operatorname{rot}(\vec{v} \times \vec{B})),(8)$ is the equation for a field with no divergence $(\operatorname{div} \vec{B}=0),(9)-(11)$ are the equation for field generation by electric currents (with no term for displacement currents, $\operatorname{rot} \vec{B}=$ $(4 \pi / c) \vec{j}),(12)$ is the Poisson's equation, (13) is the energy equation, $\vec{v}\left(v_{r}, v_{\varphi}, v_{z}\right)$ is the velocity, $\vec{B}\left(B_{r}, B_{\varphi}, B_{z}\right)$ is the magnetic field strength, $\vec{j}\left(j_{r}, j_{\varphi}, j_{z}\right)$ is the current density, $c$ is the velocity of light, $\varphi_{\mathrm{G}}$ is the gravitational potential.

Under assumption of a plane symmetry the set of equations (1)-(14) is solved for a star of mass $M$ at the following boundary conditions:

(a) $P=\rho=T=B_{\varphi}=0$ on the outer boundary,

(b) $v_{r}=j_{r}=B_{r}=0 \quad$ at $r=0$,

(c) $v_{\varphi}=j_{\varphi}=B_{\varphi}=0$ at $r=0$,

(d) $v_{z}=0, \quad \frac{\partial j_{z}}{\partial z}$ or $j_{z}=0, \quad \frac{\partial B_{z}}{\partial z}$ or $B_{z}=0$

at $z=0$.

The dissipative processes are neglected in calculations, the neutrino emission is allowed for by $f_{\nu}$, an artificial viscosity is used for shock calculations. The introduction of artificial viscosity in [2], where a Lagrangian coordinate system is used, implies replacing $P$ in the equations of motion (1)-(3) and energy (13) by

$$
P+\omega_{1}=P-\nu \operatorname{div} \vec{v}=P-\nu\left[\frac{1}{r} \frac{\partial\left(r v_{r}\right)}{\partial r}+\frac{\partial v_{z}}{\partial z}\right]
$$

where $\nu$ is the viscosity coefficient. The distributions $\rho(\vec{r}), T(\vec{r}), \vec{B}(\vec{r})$ are specified at initial time, and the last of them should satisfy the condition of the absence of magnetic charges (8) and yields finite values of $\vec{j}(\vec{r})$ throughout the star, in accordance with (9)-(11). Surface and linear currents arising from singularities in $\vec{j}(\vec{r})$ are usually ignored in calculations. If the equality (8) does hold at the beginning, it will remain valid with time provided that only Equations (5)-(7) are used to determine the field. 


\subsection{1-D Cylindrical Approximation}

A cylinder uniform along the $z$-axis with $v_{z}=B_{z}=j_{r}=j_{\varphi}=0$ is considered in a 1-D formulation. It means that in a real star the motion along the $z$-axis is neglected. The basic equations with the Lagrangian independent variable

$$
s=\int_{0}^{r} \rho^{\prime} r^{\prime} \mathrm{d} r^{\prime}
$$

become $[1,10]$

$$
\begin{aligned}
\frac{\partial}{\partial t}\left(\frac{1}{\rho}\right) & =\frac{\partial}{\partial s}\left(r v_{r}\right), \quad \frac{\partial r}{\partial t}=v_{r}, \quad \frac{\partial \varphi}{\partial t}=\frac{v_{\varphi}}{r}, \\
\frac{\partial v_{r}}{\partial t}-\frac{v_{\varphi}^{2}}{r} & =-r \frac{\partial P}{\partial s}-\frac{1}{8 \pi r} \frac{\partial}{\partial s}\left(r B_{\varphi}\right)^{2}+g, \\
\frac{\partial}{\partial t}\left(r v_{\varphi}\right) & =\frac{r B_{r}}{4 \pi} \frac{\partial}{\partial s}\left(r B_{\varphi}\right), \\
\frac{\partial}{\partial t}\left(\frac{B_{\varphi}}{\rho r}\right) & =r B_{r} \frac{\partial}{\partial s}\left(\frac{v_{\varphi}}{r}\right), \quad r B_{r}=A=\mathrm{const}, \\
\frac{\partial E}{\partial t} & =-P \frac{\partial}{\partial s}\left(r v_{r}\right)-f_{\nu}, \\
g & =-2 G \frac{2 \pi\left(M_{0}+s\right)}{r} .
\end{aligned}
$$

Here $M_{0}$ is the mass per radian of a unit length of a core with a uniform solid-body rotation, $g$ is a gravitational acceleration. An approximate equation of state in the form

$$
P=\left\{\begin{array}{c}
3.09 \times 10^{12} \rho^{5 / 3}\left(1+1.59 \times 10^{-3} \rho^{1 / 3}\right) \\
/\left(1+3.18 \times 10^{-3} \rho^{1 / 3}\right)^{2} \\
+6.5 \times 10^{4} \rho^{2}+a T^{4} / 3+\rho \mathcal{R} T \\
\text { for } \rho<3 \times 10^{9} \mathrm{~g} \mathrm{~cm}^{-3} \\
2.04 \times 10^{27}+6.5 \times 10^{4} \rho^{2}+a T^{4} / 3+\rho \mathcal{R} T \\
\text { for } \rho \geq 3 \times 10^{9} \mathrm{~g} \mathrm{~cm}^{-3}
\end{array}\right.
$$

$$
E=\left\{\begin{array}{c}
\left(4.635 \times 10^{12} \rho^{2 / 3}\right) /\left(1+3.18 \times 10^{-3} \rho^{1 / 3}\right) \\
+6.5 \times 10^{4} \rho+a T^{4} / \rho+\frac{3}{2} \mathcal{R} T \\
\text { for } \rho<3 \times 10^{9} \mathrm{~g} \mathrm{~cm}^{-3} \\
5.19 \times 10^{27} / \rho+2.41 \times 10^{18}\left(\rho-3 \times 10^{9}\right) / \rho \\
+6.5 \times 10^{4} \rho+a T^{4} / \rho+\frac{3}{2} \mathcal{R} T \\
\text { for } \rho \gg 3 \times 10^{9} \mathrm{~g} \mathrm{~cm}^{-3}
\end{array}\right.
$$

has been used in $[1,10]$. The equation allows approximately for the transition from nonrelativistic to relativistic electrons occurring at a strong degeneration. The electron pressure has been taken to be constant after the onset of neutronization. Neutrino losses due to URCA-processes has been taken into account.

At initial time $t=0$ it has been adopted $T=0$, and the density distribution has been specified in the form [1]

$$
\rho(s, 0)=a \exp \left[-b\left(r-R_{0}\right)^{2}\right] ; \quad a, b=\text { const. }
$$

Here $R_{0}=R(0, t)$ is the core radius; $M$ is the envelope mass per unit length per radian. It is adopted $v_{r}(s, 0)=0, B_{\varphi}(s, 0)=0$, and the boundary conditions (15a). Also specified are the constant $A$ from (21) and the initial distribution $v_{\varphi}(s, 0)$ from the radial equilibrium equation (19) with $\partial v_{r} /$ $\partial t=0$

$$
\frac{v_{\varphi}^{2}(s, 0)}{r(s, 0)}-r(s, 0) \frac{\partial}{\partial s} P(s, 0)+g(s)=0
$$

The angular momentum of the system core + envelope is assumed to conserve throughout the calculations; the relation for this conservation, owing to the continuity of $v_{\varphi}$ on the core boundary, is written in the form of the boundary condition

$$
\frac{M_{0}}{2} \frac{\partial h}{\partial s}-h=0 \quad \text { at } s=0, h=r B_{\varphi} .
$$




\subsection{Calculational Results in 1-D}

The problem was calculated numerically in the region

$$
t>0, \quad 0<s<M \quad\left(R_{0}<r<R(t)\right) .
$$

The basic dimensionless parameters of the problem are

$$
\alpha=\frac{A^{2}}{4 \pi M V_{0}^{2}}\left(V_{0}=\sqrt{2 \pi G M_{0}}\right), \quad \beta=\frac{M_{0}}{M} .
$$

The solution in [1] was obtained for $\beta=1, \alpha=$ $10^{-2}, 10^{-4}, 10^{-8}$. In order to introduce dimensionless quantities, all the variables are taken in the form $F=F_{0} \tilde{F}$ with the following scale variables $F_{0}$ :

$$
\begin{aligned}
& v_{0}=V_{0}, \quad r_{0}=R_{0}, \quad t_{0}=R_{0} / V_{0}, \\
& \quad h_{0}=A, \quad \rho_{0}=M / R_{0}^{2}, \\
& P_{0}=M V_{0}^{2} / R_{0}^{2}, \quad E_{0}=V_{0}^{2}, \\
& \quad \Omega_{0}=V_{0} / R_{0}\left(\Omega=v_{\varphi} / r\right), \\
& s_{0}=M, \quad T_{0}=V_{0}^{2} / 10^{3} \mathcal{R}, \quad f_{\nu 0}=V_{0}^{3} / R_{0} ; \\
& \quad \text { with } R_{0}=10^{6} \mathrm{~cm}, \quad 2 \pi M_{0}=0.5 \times 10^{-6} M \odot
\end{aligned}
$$

Decreasing the parameter $\alpha$ causes the timescales of processes to increase as $\alpha^{-1 / 2}$. As $\alpha \rightarrow 0$, it is convenient to introduce the dimensionless functions

$$
\begin{aligned}
& t_{\alpha}=t \alpha^{1 / 2}, \quad v_{r \alpha}=v_{r} \alpha^{-1 / 2}, \\
& h_{\alpha}=h \alpha^{1 / 2}, \quad f_{\nu \alpha}=f_{\nu} \alpha^{-1 / 2},
\end{aligned}
$$

having the same relationships between them for all small $\alpha$. For other functions $F_{\alpha}=F$. The results of numerical calculations are presented in Figs. 1-4 from [1].

Propagation of a slow $\left(v<v_{A}=B \sqrt{4 \pi \rho}\right)$ MHD shock over the envelope may be seen in Figs. 1 and 2. The region of the sharp temperature peak beyond the discontinuity front is the major source of neutrino emission. It is clear from Fig. 4 that the relationships between the variables (32) are little sensitive to decreasing $\alpha$. The timescale growth proportional to $\alpha^{-1 / 2}$ is caused by an increase $\sim \alpha^{-1 / 2}$ in the number of turns of magnetic lines required for achieving the condition for the onset of runaway $\epsilon_{\mathrm{M}} \sim \epsilon_{\mathrm{G}}$. Figure 3 illustates an increase in the number of turns with decreasing $\alpha$ for the same time $t_{\alpha}$. Figure 4 demonstrates the conversion of the rotational energy into other energy forms.

Estimates based on the inclusion of the spherical gravitation potential of a real star and results of numerical computations give for the mass and energy of the shed material

$$
M_{\mathrm{sh}} \approx 0.13 M_{\odot}, \quad \epsilon_{\mathrm{sh}} \approx 0.035 \epsilon_{\mathrm{rot}}
$$

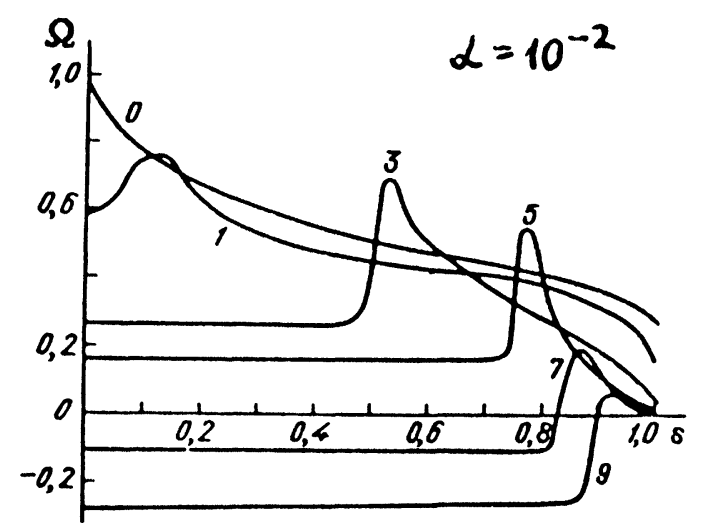

FIGURE 1 The distribution over the dimensionless parameter $s$ of the angular velocity $\Omega$ normalized to the maximum dimensionless value $\Omega^{*}=1$ at various times for $\alpha=0.01$. All curves are labeled by corresponding times $t_{\alpha}$, from [1].

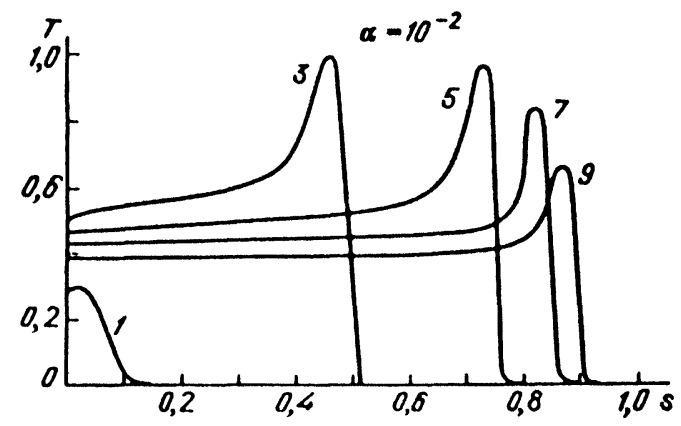

FIGURE 2 The distribution over the dimensionless parameter $s$ of the temperature $T$ normalized to the maximum dimensionless value $T^{*}=64.6$ at various times for $\alpha=0.01$. All curves are labeled by corresponding times $t_{\alpha}$, from [1]. 


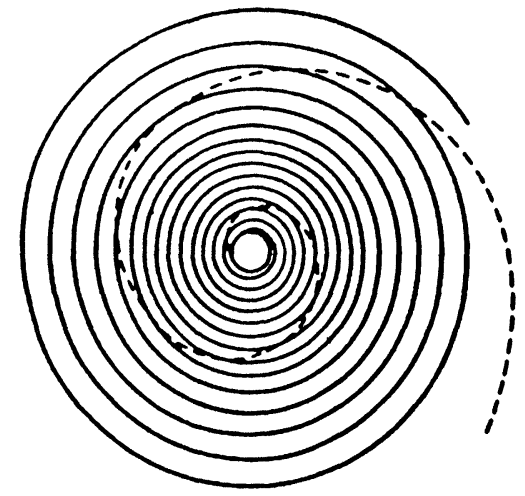

FIGURE 3 The shape of lines of magnetic force in a region nearby the core at time $t_{\alpha}=7$ for $\alpha=0.01$ (dashed line) and $\alpha=10^{-4}$ (solid line), from [1].

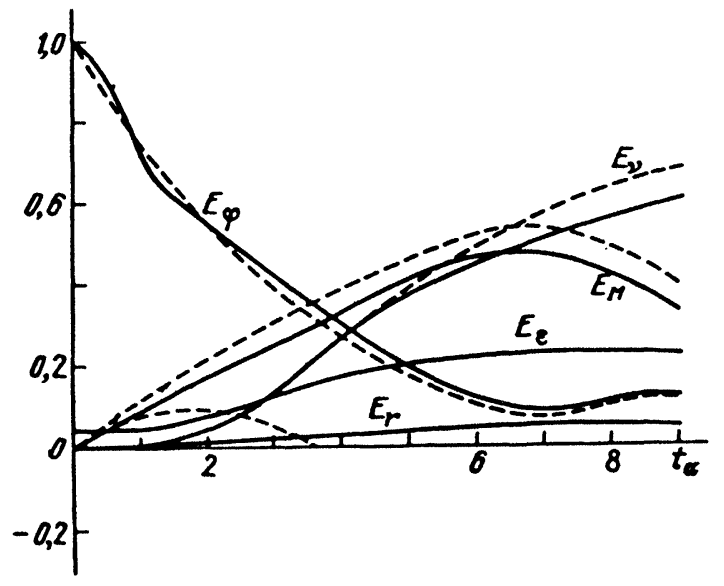

FIGURE 4 Variations with $t_{\alpha}$ of the rotational $E_{\phi}$, radial kinetic $E_{\mathrm{r}}$, internal thermal $E_{\epsilon}$, magnetis $E_{\mathrm{M}}$ energies, of the neutrino energy losses $E_{\nu}$ for $\alpha=0.01$ (solid line) and $\alpha=10^{-4}$ (dashed line). All the quantities are normalized to the maximum rotational energy $E_{\phi}^{*}=10^{47} \mathrm{erg} / \mathrm{cm}$ per unit cylinder length, from [1].

which is valid only for small $\alpha$; for $\alpha=10^{-2}$ we have $\epsilon_{\mathrm{sh}} \approx 0.08 \epsilon_{\text {rot }}$. The major part of the envelope joins the core and rotates as a rigid body together with it. The angular velocity of the resulting model is $\sim 0.1 V_{0} / R_{0}$, i.e. decreases by $\sim 10$ times relative to the initial velocity. Most of the initial rotational energy escapes in the form of neutrino emission, while most of the angular momentum is taken off by the ejected envelope. The parameter $\alpha$ has little effect on the integral neutrino flux

$$
Q_{\nu}=\int_{0}^{t} \int_{0}^{M} f_{\nu} \mathrm{d} s \mathrm{~d} t=\int_{0}^{t_{\alpha}} \int_{0}^{M} f_{\nu \alpha} \mathrm{d} s \mathrm{~d} t_{\alpha} .
$$

An interesting result of calculations is a possible stage of magnetorotational oscillations of the coreenvelope system, during which the angular velocity changes its sign. The angular velocity of the resulting core may be opposite to the initial angular velocity.

\subsection{Results of 2-D Calculations}

The attempt to obtain magnetorotational explosion (MRE) in realistic 2-D picture has been done in [3]. The simpifled problem was solved for initially uniform and rigidly rotating gas cloud and initial values of internal, rotational and magnetic energies taken as

$$
\frac{E_{\mathrm{in} 0}}{\left|E_{\mathrm{gr} 0}\right|}=0.1, \quad \frac{E_{\mathrm{rot} 0}}{\left|E_{\mathrm{gr} 0}\right|}=0.04, \quad \frac{E_{\mathrm{mag} 1}}{\left|E_{\mathrm{in} 1}\right|}=0.05 .
$$

Here the index " 0 " is related to the initial state of the collapse, index " 1 " is related to the quasistationary state, which the rotating cloud without magnetic field reaches in the process of collapse. The magnetic field was included into calculations in the point "1". This simplifies the calculations of the collapse and may be justified for a realistic case of the neutron star with $E_{\operatorname{mag} 1} / E_{\text {in } 1} \ll 1$, that it does not influence the process of the collapse. The dynamical action of the magnetic field begins to be important only after its considerable amplification in the process of twisting, which takes time much longer than the time of the collapse and establishing of the quasi-stationary state. The quadrupole component of the magnetic field is expected to be the most important for MRE, because it has large radial component near the equator, which is amplified by the field twisting. In order to avoid a central singularity a regular field of the similar topology was chosen instead of the quadrupole in 
the form (in nondimensional variables)

$$
\begin{gathered}
H_{r 1}=F_{r}(0.5 r, 0.5 z-2.5)-F_{r}(0.5 r, 0.5 z+2.5) \\
H_{\phi 1}=0 \\
H_{z 1}=F_{z}(0.5 r, 0.5 z-2.5)-F_{z}(0.5 r, 0.5 z+2.5) \\
F_{r}(r, z)=k\left(\frac{2 r z}{\left(z^{2}+1\right)^{3}}-\frac{2 r^{3} z}{\left(z^{2}+1\right)^{5}}\right), \\
F_{z}(r, z)=k\left(\frac{1}{\left(z^{2}+1\right)^{2}}-\frac{r^{2}}{\left(z^{2}+1\right)^{4}}\right)
\end{gathered}
$$

Nondimensional variables in the Equations (1)(14) have been used with a factor

$$
H_{0}=\sqrt{P_{0}}=\rho_{0}^{1 / 2} r_{0} / t_{0}
$$

for the magnetic field components. The value $k=0.43$ adjusts the energy relation (34). The numerical method is based on the generalization of the implicit Lagrangian code to the case with a magnetic field [3]. On the outer boundary a nonzero value of the pressure $P_{\text {out }}=10^{-3} P_{0}$ was kept, which have not influenced the MRE process, but solved some numerical problems. At $r=0$ it was assumed

$$
v_{r}=v_{\phi}=B_{r}=B_{\phi}=(\nabla \times \mathbf{B})_{r}=(\nabla \times \mathbf{B})_{\phi}=0
$$

and at $z=0$

$$
v_{z}=B_{z}=\frac{\partial(\nabla \times \mathbf{B})_{z}}{\partial z}=0
$$

was taken. The results of computations are presented in Figs. 5-8. The quasi-stationary state in Fig. 5 is presented at the moment very close to $t_{1}=$ 23.920, when the magnetic field (35) was included into computations. The magnetic field configuration (practically initial) at almost the same time is presented in Fig. 6. Azimuthal component of the magnetic field increases until it becomes important for a dynamical influence. Magnetic pressure pushes out the matter, mainly in the equatorial plane, which expands and part of it (about 2.4\%) is flying away to the infinity, carring away about
$0.5 \%$ of the rotational energy of the configuration, formed after the collapse. Density contours and velocity fields, showing the development of the outburst, are given Figs. 7 and 8.

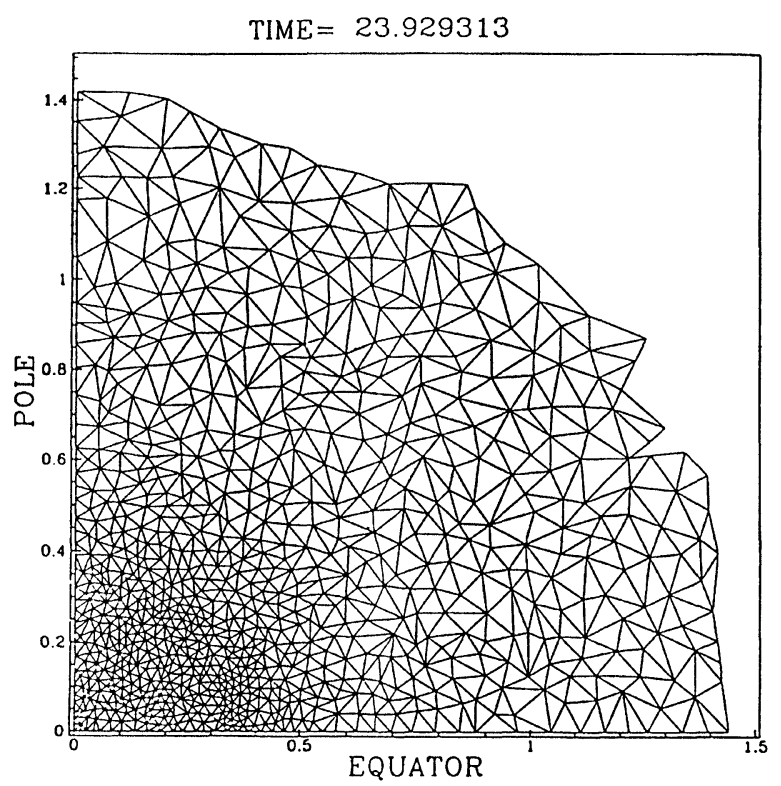

FIGURE 5 Triangular grid at $t=23.929313$, from [3].

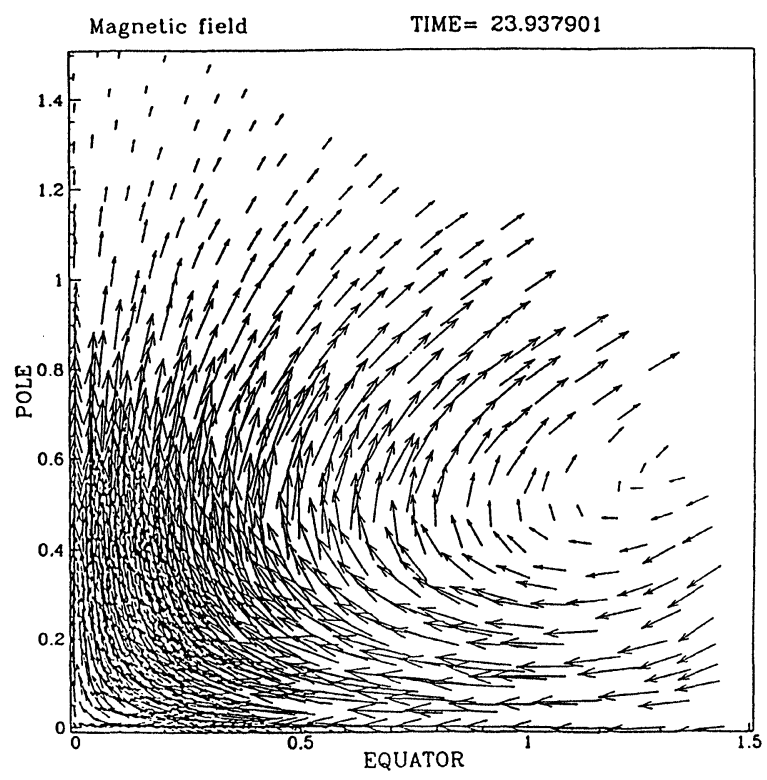

FIGURE 6 Magnetic field patterns at $t=23.94$, from [3]. 


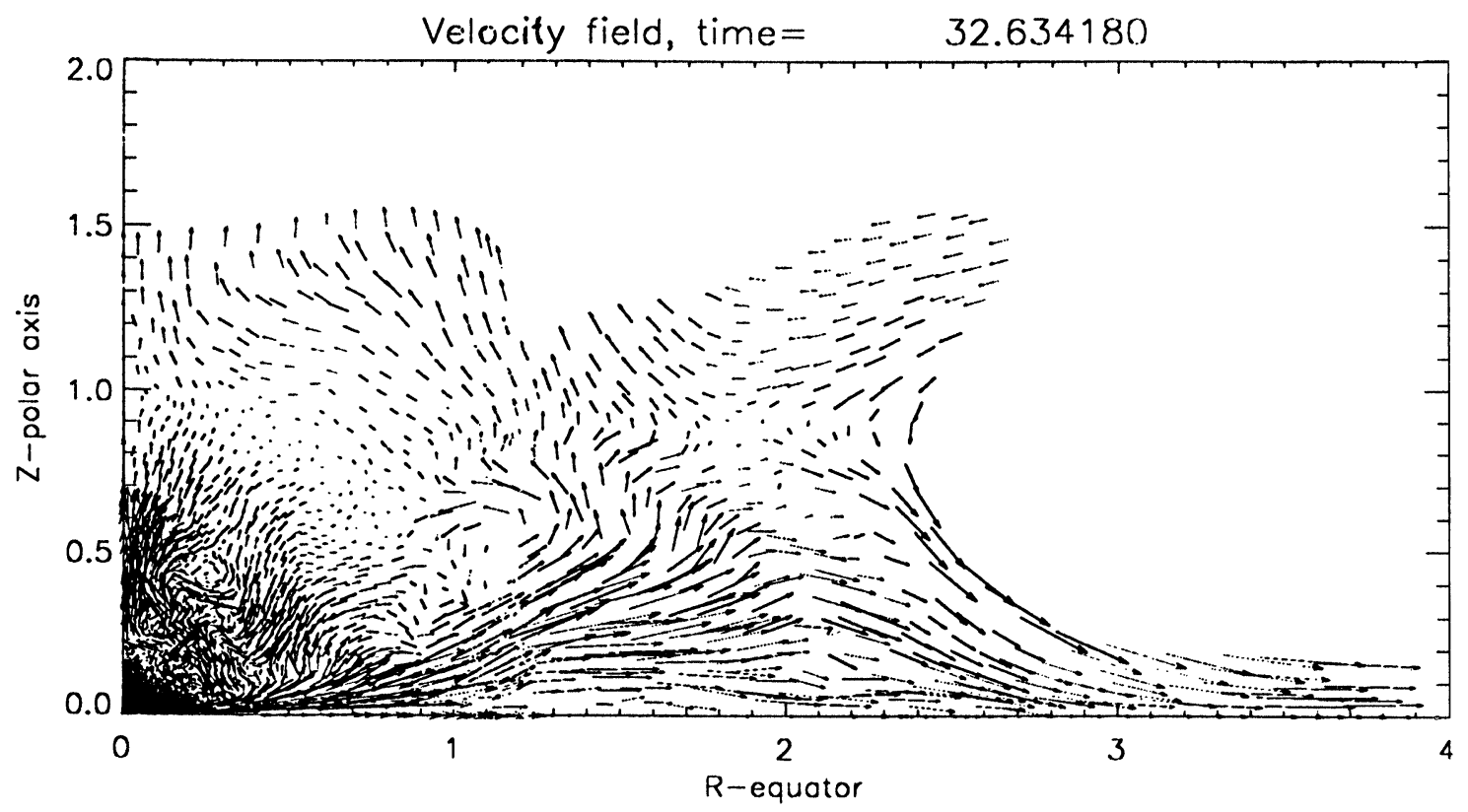

FIGURE 7 Velocity field in the meredional section of the cloud at $t=32.634$, from [4].

\section{COSEQUENCES OF A MIRROR SYMMETRY BREAKING OF THE MAGNETIC FIELD DURING COLLAPSE OF A ROTATING STAR AND NEUTRON STAR FORMATION}

Observation of the pulsars moving at the velocities up to $500 \mathrm{~km} / \mathrm{s}$ [22] is a challenge to the theory of the neutron star formation. The plausible explanation for the birth of rapidly moving pulsars seems to be the suggestion of the kick at the birth from the asymmetric explosion. Make estimations for the strength of the kick produced by the asymmetric neutrino emission during the collapse. The asymmetry of the neutrino pulse, is produced by the asymmetry of the magnetic field distribution, formed during the collapse and differential rotation.

Consider rapidly and differentially rotating new born neutron star with the dipole poloidal and symmetric toroidal fields. A field amplification during the differential rotation leads to the formation of an additional toroidal field from the poloi- dal one. This field, made from the dipole poloidal one by twisting, is antisymmetric with respect to the symmetry plane. The sum of the initial symmetric with the induced antisymmetric toroidal fields has no plane symmetry.

In absence of dissipative processes the neutron star returns to the state of rigid rotation loosing the induced toroidal field and restoring mirror symmetry of the matter distribution. Formation of asymmetric toroidal field distribution is followed by MRE, which is asymmetric, leading to neutron star recoil and star acceleration [9]. The neutron star acceleration happens also [8] due to dependence of the cross-section of week interactions on the magnetic field.

In the magnetic field electrons occupy discrete energy (Landau) levels for motion in the plane perpendicular to the field, with energy difference

$$
\Delta \epsilon_{\mathrm{L}}=\hbar \frac{e B}{m_{\mathrm{e}} c} \text {. }
$$

When this difference is of the order of the energy of beta decay $\Delta$ the decay probability begins to 


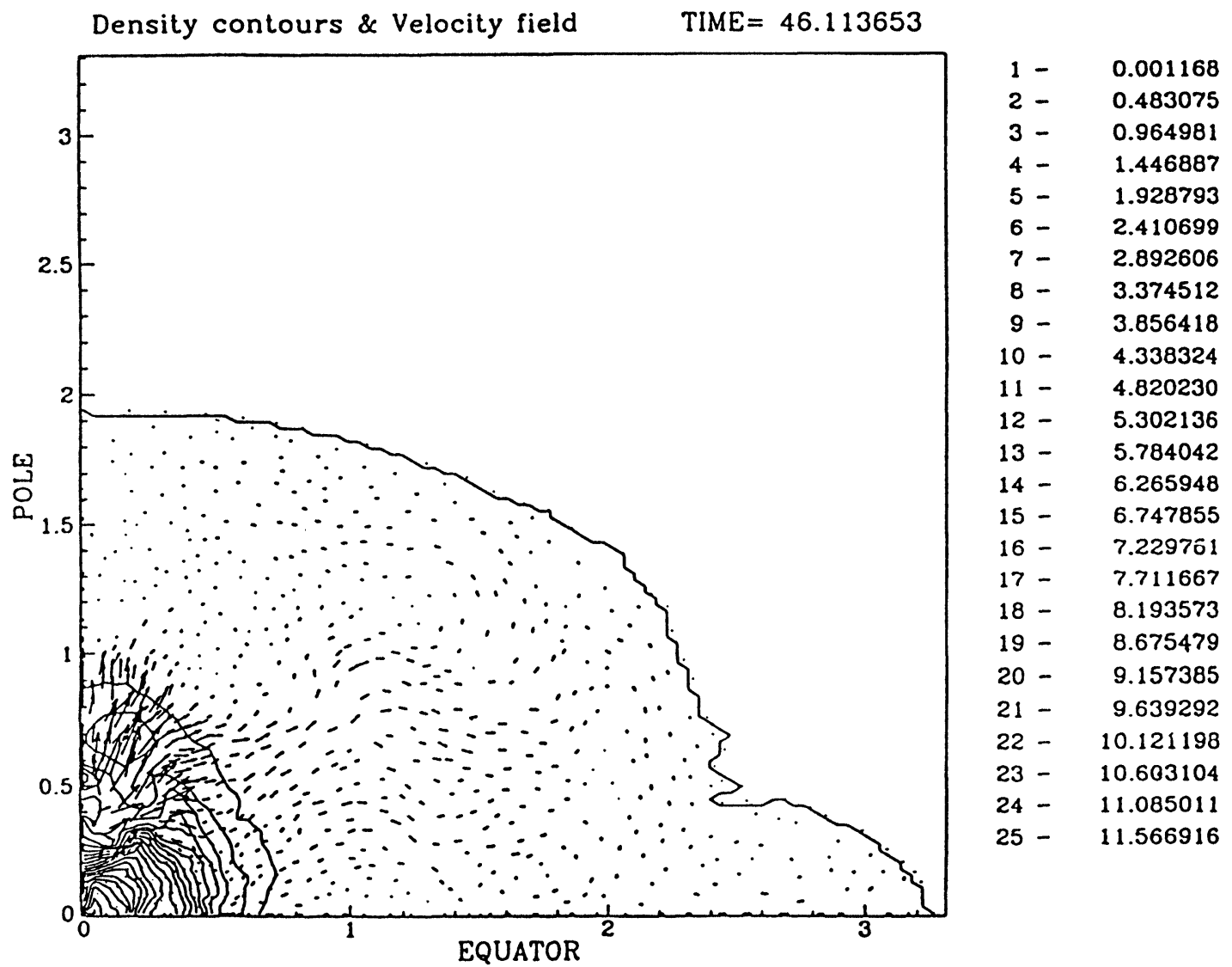

FIGURE 8 Density contours and velocity field at $t=46.11$, from [3].

depend on the value of $B$. The critical magnetic field corresponds to

$$
\Delta \epsilon_{\mathrm{L}}=\lambda m_{\mathrm{e}} c^{2}, \quad B_{\mathrm{cr}}=\lambda \frac{m_{\mathrm{e}}^{2} c^{3}}{e \hbar} \simeq 4.4 \times 10^{13} \mathrm{Gs} .
$$

The probability of the neutron decay in the strong magnetic field was calculated in [29]

$$
W_{\mathrm{n} B}=W_{\mathrm{n}}\left[1+0.17\left(\frac{B}{B_{\mathrm{cr}}}\right)^{2}+\cdots\right] \quad \text { for } B \ll B_{\mathrm{cr}}
$$

and

$$
W_{\mathrm{n} B}=W_{\mathrm{n}} 0.77\left(\frac{B}{B_{\mathrm{cr}}}\right) \text { for } B>B_{\mathrm{cr}}
$$

with $W_{\mathrm{n}}$ is the probability for nonmagnetic case, see [7]. In strongly relativistic plasma with Fermi energy $\epsilon_{\mathrm{Fe}}$ or $k T$ larger than $\Delta>m_{\mathrm{e}} c^{2}$ we should use in (40) a maximum between $\lambda=\epsilon_{\mathrm{Fe}} /\left(m_{\mathrm{e}} c^{2}\right)$ and $\lambda=k T /\left(m_{\mathrm{e}} c^{2}\right)$.

After a collapse of rapidly rotating star the neutron star rotates at the period $P$ about $1 \mathrm{~ms}$. Differential rotation leads to the linear amplification of the toroidal field

$$
B_{\phi}=B_{\phi 0}+B_{\mathrm{p}}(t / P)
$$

The time of the neutrino emission is several tens of seconds [28]. After $20 \mathrm{~s}$ the induced toroidal magnetic field will be about $2 \times 10^{4} B_{\mathrm{p}}$, corresponding to $10^{15}-10^{17} \mathrm{Gs}$ for $B_{\mathrm{p}}=10^{11}-10^{13} \mathrm{Gs}$, observed in the pulsars. Adopting the initial 
toroidal field $B_{\phi 0}=\left(10-10^{3}\right) B_{\mathrm{p}}=10^{12}-10^{16}$, we may estimate an asymmetry of the neutrino pulse. For symmetric $B_{\phi, 0}$ and dipole poloidal field the difference $\Delta B_{\phi}$ between the magnetic field's absolute values in two hemispheres increases, until it reaches the value $2 B_{\phi 0}$. It remains constant later, while the relative difference

$$
\delta_{B}=\frac{\Delta B_{\phi}}{B_{\phi+}+B_{\phi-}}
$$

decreases. The main neutrino flux is formed in the region where the mean free path of the neutrino is smaller than the stellar radius. The quantity $l_{T}$ having the meaning of the neutrino mean free path is connected with the neutrino opacity $\kappa_{\nu}$ as

$$
\kappa_{\nu}=1 /\left(l_{T \rho}\right) .
$$

Calculations of the spherically symmetrical collapse [28] have shown that during the phase of the main neutrino emission a hot neutron star consists of the quasi-uniform quasi-isothermal core with the temperature $T_{i}$, whose mass increases with time, and the region between the neutrinosphere and the isothermal core, where the temperature smoothly decreases in about 10 times, while the density, which finally drops about 6 times, decreases nonmonotonically. Neutrino flux is forming in this region, containing about one half of the neutron star mass. We suggest for simplicity a power-law dependences for the temperature and $l_{T}$ :

$$
T=T_{i}\left(\frac{r_{i}}{r}\right)^{m}, \quad l_{T}=\frac{1}{\kappa \rho}=l_{T_{i}}\left(\frac{r}{r_{i}}\right)^{n} .
$$

The neutrinosphere with the radius $r_{\nu}$ is determined approximately by the relation

$$
\int_{r_{\nu}}^{\infty} \kappa_{\nu} \rho \mathrm{d} r=\int_{r_{\nu}}^{\infty} \frac{\mathrm{d} r}{l_{T}}=1 .
$$

Using (46) outside the neutrinosphere we get from (47) the relation

$$
r_{\nu}=r_{i}\left(\frac{r_{i}}{(n-1) l_{T_{i}}}\right)^{1 /(n-1)} .
$$

Finally we get the temperature of the neutrinosphere $T_{\nu}$ and the neutrino luminosity $L_{\nu}$

$$
\begin{aligned}
T_{\nu}= & T_{i}\left(\frac{(n-1) l_{T_{i}}}{r_{i}}\right)^{m /(n-1)} \\
L_{\nu}=4 \pi r_{\nu}^{2} H_{\nu}= & \frac{7}{8} m \frac{16 \pi a c T_{i}^{4}}{3}(n-1)^{(4 m-n+1) /(n-1)} \\
& \times r_{i}^{2}\left(\frac{l_{T_{i}}}{r_{i}}\right)^{(4 m-2) /(n-1)}
\end{aligned}
$$

To estimate the anisotropy of the neutrino flux we compare two stars with the same radius and temperature of the core $r_{i}$ and $T_{i}$ and different opacities. Let $l_{T_{i}}$ is different and constant in two hemispheres, and each one is radiating according to (50). The anisotropy of the flux

$$
\delta_{L}=\frac{L_{+}-L_{-}}{L_{+}+L_{-}},
$$

here $L_{+}, L_{-}$are luminosities in the different hemispheres, calculated using (50). For small difference between hemispheres

$$
\delta_{L}=\frac{\Delta L}{L}=\frac{4 m-2}{n-1} \frac{\Delta l_{T_{i}}}{l_{T_{i}}} .
$$

Here $n>1$, when $m=\frac{1}{2}$ the neutrino fluxes in both hemispheres are equal because smaller opacity and larger neutrinosphere temperature $T_{\nu}$ from (49) is compensated by smaller neutrinosphere radius $r_{\nu}$ from (48). The equation of motion of the neutron star with the mass $M_{\mathrm{n}}$

$$
M_{\mathrm{n}} \frac{\mathrm{d} v_{\mathrm{n}}}{\mathrm{d} t}=\frac{L_{+}-L_{-}}{c}, \quad L_{+}+L_{-}=\frac{2}{\pi} L_{\nu}(t) .
$$

For the power distributions it follows from (50) that

$$
L_{ \pm}=A l_{T_{i} \pm}^{(4 m-2) /(n-1)} .
$$

As an example consider the dependence on $B$ in the form (41) and (42). Making interpolation 
between two asymptotic forms we get dependence

$$
\begin{aligned}
l_{T_{i} \pm} \sim \frac{1}{W} & =l_{T_{0}} \frac{1+\left(B / B_{\mathrm{cr}}\right)^{3}}{1+0.17\left(B / B_{\mathrm{cr}}\right)^{2}+0.77\left(B / B_{\mathrm{cr}}\right)^{4}} \\
& =l_{T_{0}} F^{(n-1) /(4 m-2)}(B) .
\end{aligned}
$$

The time dependence of the average value of $B$ in each hemisphere can be found from (43) with

$$
B_{\mathrm{p}+}=-B_{\mathrm{p}-}, \quad B_{\phi 0+}=B_{\phi 0-} .
$$

By $B_{\mathrm{p}}$ we mean a radial component of the poloidal field taking part in amplification of $B_{\phi}$. The time dependence of $L_{\nu}$ is taken from the spherically symmetric calculations of the collapse.

For $(4 m-2) /(n-1)=1$ and in condition when the neutron star is accelerated at $B \gg B_{\mathrm{cr}}$, we have $F_{ \pm}=B_{\mathrm{cr}} / 0.77 B_{ \pm}$. Equation of motion (53) may be written as

$$
M_{\mathrm{n}} \frac{\mathrm{d} v_{\mathrm{n}}}{\mathrm{d} t}=\frac{2}{\pi} \frac{L_{\nu}}{c} \frac{\left|B_{+}\right|-\left|B_{-}\right|}{\left|B_{+}\right|+\left|B_{-}\right|}
$$

with the linear functions for $B_{ \pm}$. Take constant $L_{\nu}=0.1 M_{\mathrm{n}} c^{2} / 20 s$. With these simplifications, the final velocity of the neutron star $v_{\mathrm{nf}}$ follows as a result of the solution of (57) in the form

$$
v_{\mathrm{nf}}=\frac{2}{\pi} \frac{L_{\nu}}{M_{\mathrm{n}} c} \frac{P B_{\phi 0}}{\left|B_{\mathrm{p}}\right|}\left(0.5+\ln \left(\frac{20 s}{P} \frac{\left|B_{\mathrm{p}}\right|}{B_{\phi 0}}\right)\right) .
$$

For $P=10^{-3}$ s we obtain

$$
\begin{aligned}
v_{\mathrm{nf}} & =\frac{2}{\pi} \frac{c}{10} \frac{P}{20 s} x\left(0.5+\ln \left(\frac{20 s}{P} \frac{1}{x}\right)\right) \\
& \approx 1 \frac{\mathrm{km}}{\mathrm{s}} x\left(0.5+\ln \frac{2 \times 10^{4}}{x}\right) .
\end{aligned}
$$

For the value $x=B_{\phi 0} /\left|B_{\mathrm{p}}\right|$ ranging between 20 and $10^{3}$, we have $v_{\mathrm{nf}}$ between 140 and $3000 \mathrm{~km} / \mathrm{s}$, what can explain the nature of the most rapidly moving pulsars. The formula (59) can be applied when $B_{\phi 0} \gg B_{\mathrm{c}}$ and $x \gg 1$.

The acceleration of the collapsing star by anisotripic neutrino emission can happen even when the star collapses to the black hole, the efficiency of acceleration decreases with increasing of mass. We may expect black holes of stellar origin moving rapidly, like radiopulsars, and they may be found high over the galactic disk. This is observed among the soft X-ray novae - most probable candidates for black holes in the galaxy.

\section{DISCUSSION}

2-D calculations of the magnetorotational model of explosion give encouraging results, showing a sufficiently high efficiency of transformation of the rotational energy into the energy of explosion to explain SN events with a formation of neutron stars. There is a question, however, will this conclusion remain valid in the real case where instabilities connected with deviations of an axial symmetry would be able to develop. The answer could be obtained from 3-D calculations, which are still not available. Qualitative estimations show that 3-D perturbations should not change drastically the results of 2-D calculations. Usually, the development of MHD perturbations happens, when magnetic pressure is larger then the gaseous one. In these situation the dynamical processes in the star, connected with hydrodynamical motion and formation of the shock wave, are developing more rapidly than possible instabilities, which increment is usually less than the characteristic time of shock wave formation and propagation. Another process which was not included in calculations is connected with final resistivity and magnetic field dissipation. Without instabilities dissipation is extremely slow because of very high conductivity of high density and high temperature plasma. Even in the case of development of small scale 3-D instabilities there is a mechanism of their nonlinear suppression, because increase of temperature diminishes the ratio of the magnetic to gas pressure and damps the instability.

These qualitative speculations should be checked by $3-D$ calculations, what seem to be a very difficult task even for modern supercomputers. It is 
connected with a necessity to use a Lagrangian numerical scheme for obtaining a reliable result in the problem of collapse and explosion in MHD, for which 3-D generalization is very nontrivial. To get a reliable result in Eulerian schemes the number of grid point should be increased to that extends which exceed not only modern but also nearest future computer abilities. Another shortcoming of Eulerian scheme lay in strong restriction of the time step. In the magnetorotational model the problem has two very different time scales, connected with a hydrodynamical wave propagation and much longer time of the magnetic field amplification, and the time step is connected with a smaller characteristic time. In this situation for realistic values of the initial magnetic field the necessary number of time steps would be so large, that even sufficiently powerful computer would not be able to give a reliable result because of the loss of a precision of any modern numerical scheme when the number of time steps exceed $\sim 10^{5}$. So, the only way to make a step to 3-D calculations of magnetorotational model is to develop an appropriate method based on a Lagrangian scheme of computing.

\section{References}

[1] N.V. Ardelyan, G.S. Bisnovatyi-Kogan and Yu.P. Popov (1979) Astron. Zh. 56, 1244.

[2] N.V. Ardelyan, G.S. Bisnovatyi-Kogan, Yu.P. Popov and S.V. Chernigovskii (1987) Astron. Zh. 64, 495

[3] N.V. Ardeljan, G.S. Bisnovatyi-Kogan and S.G. Moiseenko (1996) Ap. Space Sci. 239, 1.

[4] N.V. Ardeljan, G.S. Bisnovatyi-Kogan and S.G. Moiseenko (1997) Uspekhi Fiz. Nauk 167, 1128.

[5] D. Arnett (1967) Canad. J. Phys. 45, 1621.
[6] G.S. Bisnovatyi-Kogan (1970) Astron. Zh. 47, 813.

[7] G.S. Bisnovatyi-Kogan (1989) Physical Problems of Theory of Stellar Evolution (M. Nauka).

[8] G.S. Bisnovatyi-Kogan (1993) Astron. Ap. Transact. 3, 287.

[9] G.S. Bisnovatyi-Kogan and S.G. Moiseenko (1992) Sov. Astron. 36, 285.

[10] G.S. Bisnovatyi-Kogan, Yu.P. Popov and A.A. Samochin (1976) Ap. Space Sci. 41, 321.

[11] S.I. Blinnikov, T.A. Lozinskaya and N.N. Chugai (1987) Itogi Nauki i Tekhniki, Astronomiya 32, 142.

[12] A. Burrows and B.A. Fryxell (1993) Ap. J. Lett. 418, L33.

[13] A. Burrows, J. Hayes and B.A. Fryxell (1995) Ap. J. 450, 830.

[14] S. Colgate and R. White (1966) Ap. J. 143, 626

[15] M. Herant, W. Benz, W.R. Hix, C.L. Fryer and S.A. Colgate (1994) Ap. J. 435, 339.

[16] W. Hillebrandt (1985) Proc. NATO-ASI High Energy Phenomena Around Collapsed Stars, Cargese.

[17] V.S. Imshennik and D.K. Nadyozhin (1982) Itogi Nauki i Tekhniki, Astronomiya 21, 63.

[18] L.N. Ivanova, V.S. Imshennik and D.K. Nadyozhin (1969) Nauch. Inf., Issue 13, 3.

[19] H.-T. Janka and E. Müller (1994) Astron. Ap. 290, 496.

[20] H.-T. Janka and E. Müller (1995) Ap. J. Lett. 448, L109.

[21] L.M. Le Blank and J.R. Wilson (1970) Ap. J. 161, 541.

[22] A.G. Lyne and D.R. Lorimer (1994) Nature 369, 127.

[23] R. Mayle, J.R. Wilson and D.N. Schramm (1987) Ap. J. 318, 288.

[24] E. Müller and W. Hillebrandt (1979) Astron. Ap. 80, 147.

[25] D.S. Miller, J.R. Wilson and R.W. Mayle (1993) Ap. J. 415 , 278

[26] D.K. Nadyozhin (1977) Ap. Space Sci. 49, 399.

[27] D.K. Nadyozhin (1977) Ap. Space Sci. 51, 284.

[28] D.K. Nadyozhin (1978) Ap. Space Sci. 53, 131.

[29] R.F. O'Connell and J.J. Matese (1969) Nature 222, 649.

[30] T. Ohnishi (1983) Tech. Rep. Inst. At. En. Kyoto Univ. No. 198.

[31] M. Schwarzschild (1958): Structure and Evolution of the Stars (Princeton University Press, Princeton).

[32] T. Shimizu, S. Yamada and K. Sato (1994) Ap. J. Lett. 432, L119.

[33] I.S. Shklovski (1976): Supernovae Stars (M., Nauka).

[34] Supernova 1987A, in Proc. Workshop ESO, July 1987.

[35] J. Van den Hulst et al. (1983) Nature 306, 566.

[36] J.R. Wilson, R. Mayle, S. Woosley and T. Weaver (1986) Ann. N-Y Acad. Sci. 470, 267.

[37] S. Woosley and T. Weaver (1986) in Nucleosynthesis and its Implications on Nuclear and Particle Physics, Eds. J. Audouze and N. Mathieu (D. Reidel), p. 145. 


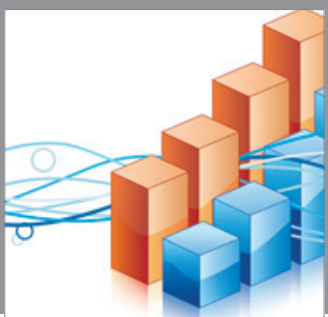

Advances in

Operations Research

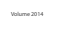

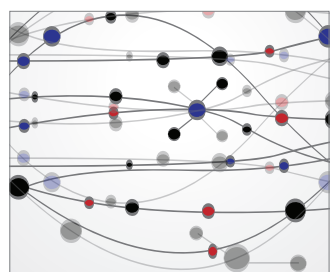

\section{The Scientific} World Journal
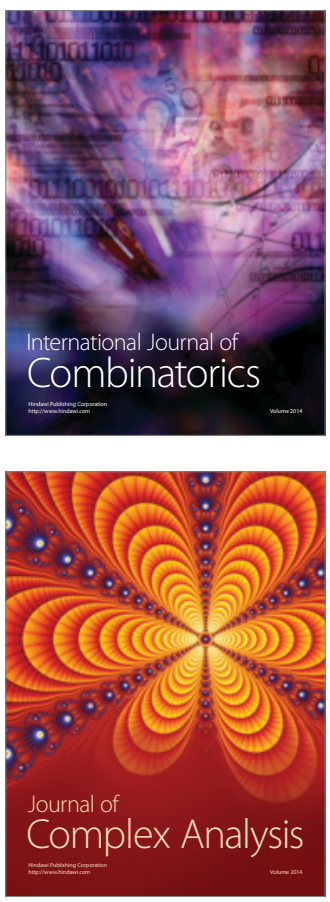

International Journal of

Mathematics and

Mathematical

Sciences
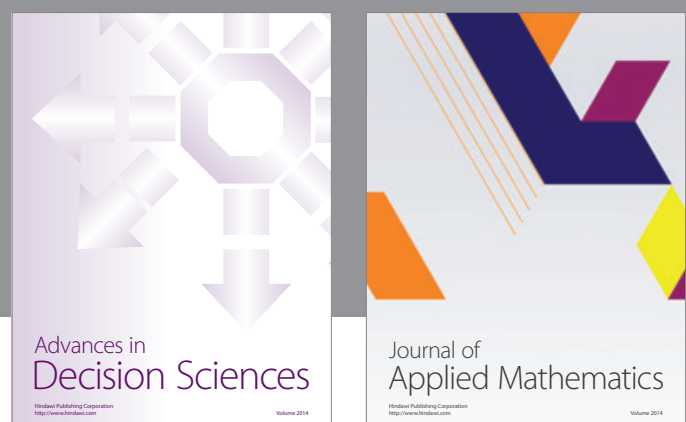

Journal of

Applied Mathematics
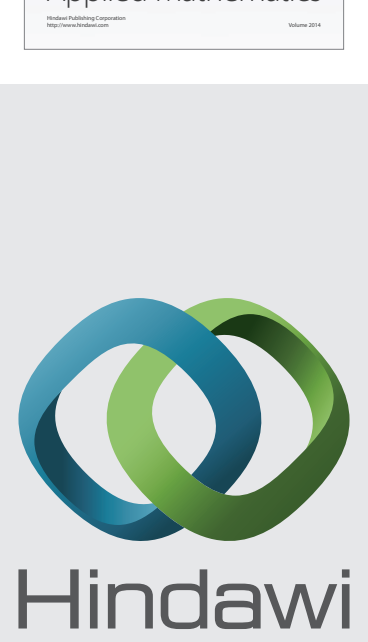

Submit your manuscripts at http://www.hindawi.com
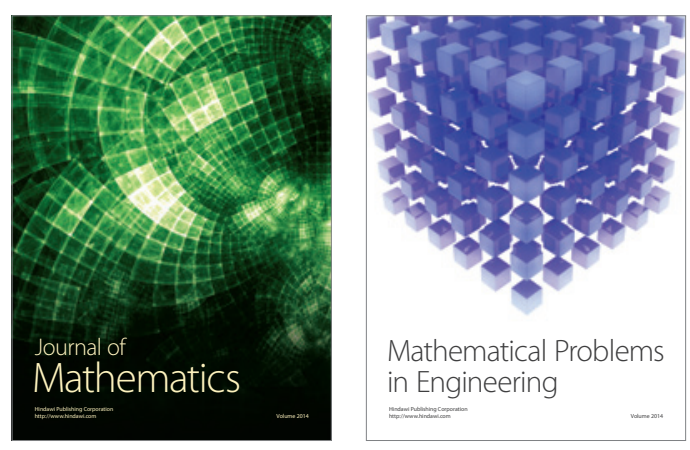

Mathematical Problems in Engineering
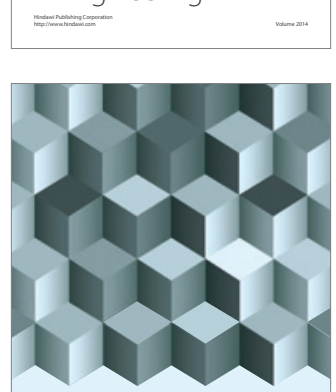

Journal of

Function Spaces
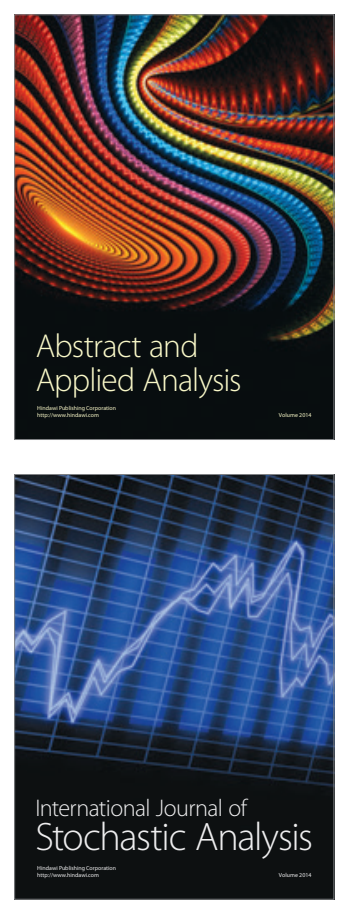

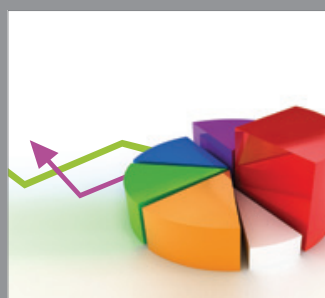

ournal of

Probability and Statistics

Promensencen
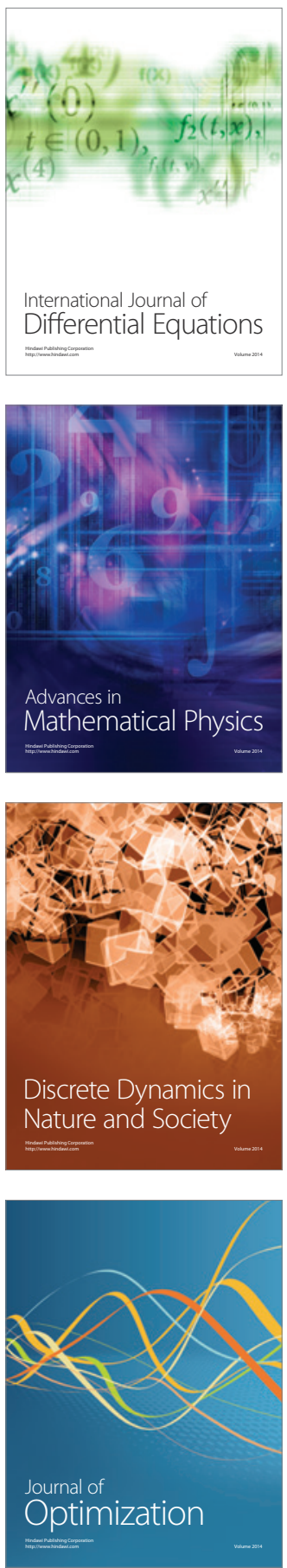